\title{
Filigrane
}

Écoutes psychothérapiques

\section{Interpréter : un processus psychique et un acte complexes}

\section{Nathalie Zilkha}

Volume 15, numéro 1, printemps 2006

URI : https://id.erudit.org/iderudit/013529ar

DOI : https://doi.org/10.7202/013529ar

Aller au sommaire du numéro

Éditeur(s)

Revue Santé mentale au Québec

ISSN

1192-1412 (imprimé)

1911-4656 (numérique)

Découvrir la revue

Citer cet article

Zilkha, N. (2006). Interpréter : un processus psychique et un acte complexes. Filigrane, 15(1), 57-66. https://doi.org/10.7202/013529ar

\section{Résumé de l'article}

Dans l'interprétation convergent un travail psychique et un acte. Son enjeu varie. Ainsi en témoigne la forme que choisit de lui donner l'analyste. Celle-ci dépend du travail psychique qu'il pense le plus utile de privilégier : déploiement de la réalité psychique et du transfert, dégagement, renoncement, deuil... Des séquences cliniques tirées de cures où l'interprétation du transfert n'a longtemps pas été de soi permettent à l'auteur de réfléchir notamment à la pertinence et aux limites d'interventions qui peuvent être qualifiées d'ouverture ou de mise en jeu. 


\title{
Interpréter : un processus psychique et un acte complexes
}

\author{
nathalie zilkha
}

\begin{abstract}
Dans l'interprétation convergent un travail psychique et un acte. Son enjeu varie. Ainsi en témoigne la forme que choisit de lui donner l'analyste. Celle-ci dépend du travail psychique qu'il pense le plus utile de privilégier : déploiement de la réalité psychique et du transfert, dégagement, renoncement, deuil... Des séquences cliniques tirées de cures où l'interprétation du transfert n'a longtemps pas été de soi permettent à l'auteur de réfléchir notamment à la pertinence et aux limites d'interventions qui peuvent être qualifiées d'ouverture ou de mise en jeu.
\end{abstract}

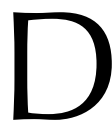

ans sa forme très secondarisée et quelque peu austère mon titre reflète l'embarras dans lequel cette réflexion sur l'interprétation m'a plongée.

Comment rendre compte des mouvements subtils entre processus primaires et processus secondaires tels qu'ils se développent dans l'intimité de la séance et se mettent assez naturellement en jeu dans l'interprétation? Et surtout, comment penser l'interprétation, ce pilier du travail analytique, sans être rattrapé par ses idéaux, individuels ou groupaux, qu'on espérait en partie dépassés ? Pour Didier Anzieu par exemple,

«il apparaît ainsi, on le sait, que la "bonne" interprétation doit être exacte, opportune, mesurée, dense, claire, concise, concrète, vivante sans être séduisante, suggestive plutôt qu'exhaustive. » $(1970,756)$

De quoi inhiber celui ou celle qui s'aventurerait sur ce sujet! Anzieu ajoute toutefois :

«[...] encore qu'il reste à chaque thérapeute de réinventer pour son propre compte la "bonne" interprétation tout au long de sa pratique. » (Anzieu, 1970,756)

À chacun donc de se situer entre la rigueur indispensable et le style propre toujours à créer.

Après quelques considérations générales, je centrerai mon propos sur l'enjeu de diverses formes d'interprétation dans les situations où la névrose de transfert, parce qu'insuffisamment organisée, est difficilement interprétable. 


\section{L'interprétation : du dire et du faire?}

Je propose de considérer que, pour chacun des protagonistes de la situation psychanalytique, l'interprétation s'inscrit à la fois dans le processus psychique et dans l'acte. L'analysant reçoit l'interprétation à ces niveaux différents, celui de l'acte et celui du contenu. Il lui arrive ainsi d'interpréter la prise de parole de l'analyste comme une séduction, une castration, une pénétration, une critique, une aliénation ou un rejet... et cela quel que soit le contenu énoncé ! Il va de soi que la signification que l'analysant donne à l'interprétation et «l'utilisation », selon l'expression de Winnicott, qu'il peut en faire dépendent de sa réalité psychique et de la configuration transférentielle précise. De plus, comme cela a été amplement discuté, le travail psychique de l'analysant est inévitablement rendu d'autant plus complexe que des mouvements contre-transférentiels inconscients infiltrent le silence de l'analyste ou ses interprétations, tant l'acte d'énonciation que l'énoncé. J'y ajouterais la qualité de l'articulation entre l'acte d'énonciation et l'énoncé, également déterminante pour l'utilisation de l'interprétation par l'analysant.

Dans les situations favorables le transfert de l'analysant sur la prise de parole de l'analyste semble se suspendre. La prise de parole de l'analyste paraît se fondre dans l'enjeu de la mise en sens, se dissoudre derrière le contenu de l'interprétation ou encore se faire silencieuse par rapport à l'investissement que l'analysant fait de l'énoncé. Ainsi, l'interprétation peut être particulièrement efficiente. Je fais là une analogie avec la présence silencieuse du cadre (Green, 1990; Donnet, 1995), lorsque la névrose de transfert s'organise suffisamment bien, qu'elle est «opérante » et donc interprétable. A contrario, lorsque l'analysant surinvestit la prise de parole de l'analyste au détriment de l'énoncé, le travail psychique achoppe facilement. «C'lui qui dit, c'est c'lui qui est !» hurlait par exemple un enfant persécuté chaque fois que je voulais intervenir. Nous pourrions aussi évoquer à ce propos une «dé-transitionnalisation» ou une «dé-symbolisation» de l'interprétation où le dire devient du «faire».

\section{De la diversité des enjeux de l'interprétation}

Il est impossible de parler d'interprétation psychanalytique sans engager la question du transfert. Mais la relation entre ces deux termes est complexe, parfois même paradoxale. Ainsi, si toute interprétation s'origine dans le transfert et s'appuie même, en partie, sur lui, elle permet, à terme, de dégager l'analysant de ce même transfert. Dit autrement, le travail d'interprétation ouvre la voie du renoncement à l'objet de transfert; de fait ce travail trouve son efficience maximale quand il peut tout à la fois s'ancrer dans le transfert et s'appuyer sur distinction entre l'objet de transfert et l'analyste dans sa fonction d'analyste.

$\mathrm{Au}$ cours du travail analytique, les modes et les chemins du renoncement, ouverts par l'interprétation, varient. Chaque interprétation ponctue en effet de manière singulière le travail analytique, chacune fait subtilement jouer la dialectique processus primaire/processus secondaire, ainsi que celle de l'identité de perception et de l'identité de pensée. Chacune comporte aussi des limites et des 
risques spécifiques et dépend de ce que peut en «faire» l'analysant. Comme nous le rappelle Jean-Luc Donnet,

«toutes les interventions interprétatives de l'analyste n'ont pas le même enjeu. Celui-ci est fonction du "matériel" concerné (affects et représentations), du niveau d'angoisse, et bien sûr du moment processuel avec ses implications transférentielles et contre-transférentielles. [...] De manière générale l'enjeu dépend de la place que l'analyste occupe dans le transfert au moment où il profère l'interprétation. » $(1995,175)$

L'enjeu de l'interprétation dépend aussi, selon moi, de la manière dont coexistent pour l'analysant, dans l'expérience du transfert, l'analyste comme objet fantasmatique et l'analyste comme objet «externe», c'est-à-dire dans sa fonction d'analyste. L'analysant les confond-il ? Les superpose-il ? Ou sont-ils bien différenciés pour lui ? Cela nous amène à considérer la rencontre entre perception et représentation dans l'expérience du transfert, ainsi que l'écart qui soutient cette rencontre. Corrélativement se pose la question de savoir quel écart l'analyste pense le plus utile de promouvoir, à ce moment précis, pour le processus. L'analyste choisira-t-il par exemple de soutenir la mise en jeu par l'analysant de sa réalité psychique? D'en encourager le déploiement? Ou bien estimera-t-il plus utile de favoriser un mouvement de dégagement de la représentation, une «désaliénation » (Donnet, 1995) voire un travail de renoncement et de deuil?

Relisant avec ces préoccupations à l'esprit le travail magistral de James Strachey (1934) La nature de l'action thérapeutique de la psychanalyse, j'ai noté l'attention qu'il porte tout au long de son développement aux conditions de possibilité d'une interprétation mutative. Ainsi Strachey met l'emphase sur la nécessité que l'analysant puisse différencier, grâce à l'interprétation, l'analyste dans sa fonction d'analyste de l'analyste comme objet fantasmatique au moment même où les mouvements pulsionnels à l'égard de l'analyste sont reconnus par l'analysant, c'est-à-dire à l'acmé de l'expérience dans le transfert :

«Dans la seconde phase d'une interprétation complète un rôle crucial revient donc au sens de la réalité du patient: l'heureuse issue de cette phase dépend en effet de son aptitude, au moment critique de l'émergence à la conscience du quantum d'énergie pulsionnelle libérée, à distinguer entre son objet fantasmatique et l'analyste. » (Strachey, 1934, 51)

Pour approfondir ces questions je me propose de développer des séquences de deux cures dans lesquelles l'interprétation du transfert n'a longtemps pas été de soi. Longtemps elle m'a semblé inutilisable par ces analysants, voire à risque de renforcer leur résistance. Dans ces situations j'ai eu à cœur de ne pas engager 
d'emblée toute la mise et, tout en restant dans «l'arène du transfert » (Freud, 1914) j'ai privilégié, dans un premier temps, des interventions qui se voulaient davantage soutenir le déploiement de leur réalité psychique qu'un mouvement de renoncement.

Il est bien entendu artificiel et hasardeux de dégager une interprétation et «l'utilisation» (Winnicott, 1971) qu'en fait le patient de l'ensemble du réseau associatif et interprétatif, complexe et dynamique, dans lequel cette interprétation trouve sa place mais ce biais est inévitable. De plus, si toute intervention s'articule à un ensemble dans lequel elle s'intègre, chacune porte son enjeu propre.

\section{La mère lionne}

Cette première illustration permettra de développer la question de l'interprétation au point d'actualisation transférentielle. Jessica est une femme dans la cinquantaine, mariée sans enfant, dans sa troisième année d'analyse. L'une des difficultés rencontrées dans la cure a été sa difficulté à utiliser toutes les séances, et la séance dans son entièreté, par peur de mon emprise et/ou de mon rejet. Il lui a notamment longtemps été «impensable» de prendre en considération mes dates de vacances pour organiser les siennes.

Lors d'une séance de reprise après mes vacances, Jessica arrive avec un retard d'un quart d'heure et me raconte un rêve qu'elle a fait au petit matin. Je note qu'elle me parle comme s'il n'y avait pas eu de séparation. Le rêve est riche mais peu condensé. Son récit est donc très long et je ne peux en rendre compte que très schématiquement. Dans la première partie de son rêve, Jessica est victime d'un vol dans sa maison alors qu'elle se trouve devant la porte de celle-ci et qu'elle regarde deux fillettes faire du patin à roulettes. Les deux policiers qu'elle rencontre dans la rue et auxquels elle se plaint semblent peu intéressés par son histoire: «Vous étiez là, lui disent-ils, l'entrée n'a pas été forcée. » Elle constate qu'il ne lui reste que des habits qui évoquent des déguisements. Dans la deuxième partie de son rêve la maison est en ruines. De l'extérieur elle aperçoit une lionne à l'intérieur qui pourrait menacer une petite fille que la patiente tente de prévenir. Puis l'extérieur et l'intérieur se confondent. Il est alors question que Jessica prenne dans les bras un lionceau mais elle se méfie de la mère lionne.

Toutes les associations de l'analysante autour de son rêve sont intéressantes mais je ne peux les reprendre toutes. Elle se dit appartenir à une famille vide d'enfants. Elle enchaîne sur la jalousie de sa cousine lorsqu'elle s'approche de ses enfants puis son sentiment qu'elle-même ne se retrouve qu'avec un semblant de vie et elle se sent déguisée. Elle se souvient alors que, dans le rêve, les lionceaux voulaient jouer avant que leur mère ne se réveille : «Même les lionceaux voulaient s'échapper de leur mère qui se réveille. » Dans le même souffle elle poursuit: «Ma mère a téléphoné hier soir, mon père le soir d'avant, je ne veux plus être collée à eux. Quand ma mère a téléphoné hier soir pour papoter je lui ai raccroché au nez. Je sens leur présence qui me capture.» Elle ajoute : «la mère lionne doit continuer à dormir. » En restant très proche du récit du rêve tel que l'analysante se le 
remémore dans le contexte transférentiel de la séance, je lui propose alors : «Pour que vous puissiez continuer à jouer, en cachette...»

Parallèlement au discours de la séance, je voudrais tenter de rendre compte des pensées qui me sont venues pendant la séance. Paradoxalement la référence transférentielle ne m'apparaît claire qu'au moment où je me mets à parler. Je me trouve, me dis-je, dans la position de la lionne dangereuse qu'il faut endormir, l'objet maternel inconscient auquel elle voudrait voler les lionceaux, c'est à dire les enfants mais plus globalement la vie et la créativité. La première partie du rêve masquerait alors, en le retournant, le désir exprimé dans le second. Il figurerait aussi son sentiment d'être vide à son tour, sans véritable identité, et sa crainte de ne pas pouvoir jouer. Je note la place qu'elle accorde au jeu dans le rêve et cela m'évoque le peu d'espace de jeu dans l'analyse. C'est ce terrain là que consciemment je veux privilégier par mon intervention et sur ce terrain là que j'interviens. Le fantasme de payer, en manque d'enfant et en vide, son désir de voler à sa mère son intérieur ne me paraît en revanche pas suffisamment proche de la conscience pour l'aborder.

Lorsque, à la suite de son «la mère lionne doit continuer à dormir», j'énonce «pour que vous puissiez continuer à jouer... en cachette...», je pense qu'une partie de la séance s'est passée en mon absence, en cachette en quelque sorte. Je suis toutefois un peu étonnée de mon intervention, elle m'a en partie échappée, j'aurais pu par exemple davantage insister sur la solitude («jouer, mais seule»). Si le «en cachette» reprend les thèmes développés au cours de séances antérieures, il reflète en effet aussi mon irritation face à une absence prochaine qu'elle m'a annoncée.

Suite à mon intervention, Jessica me dit qu'elle commence à résister à ses parents ce qu'elle trouve bien : «Je vais lutter pour ne plus être engloutie par eux. » J'entends la référence transférentielle et je choisis d'attendre. Je me dis d'une part, elle vient de m'informer qu'elle résiste. Par ailleurs, l'enjeu inconscient qui me semble motiver son rêve, son retard ainsi que son absence à venir ne me paraît pas encore suffisamment actualisé pour que mon interprétation puisse rencontrer en elle un sentiment de «vérité psychique ». Même si nous approchons de la fin de la séance, je veux éviter d'alimenter ses résistances par une interprétation inopportune; je crains surtout de renforcer des mouvements d'intellectualisation ou de mise à distance des affects. Il me vient alors que comme une lionne je suis à l'affût d'une «bonne proie», un mouvement inconscient que je pourrais interpréter. D'autant plus je viens de m'atteler à l'écriture de ce travail. J'en conclus que les conditions ne sont décidément pas réunies pour interpréter; avec un peu de tristesse je me dis même que j'y renonce... des pensées assez inhabituelles. C'est alors, et ce n'est pas le moindre des paradoxes, que s'actualise l'enjeu transférentiel d'une manière qui me paraît alors clairement interprétable.

Jessica regarde sa montre et me dit que cela ne vaut pas la peine de poursuivre puisqu'il ne nous reste qu'une minute. En fait, elle se trompe, il nous en reste cinq de plus. Elle emploie une expression usuelle «we are running out of time» qui 
contient le verbe «to run», courir ${ }^{1}$. En désignant sa montre qui, je le vois alors, indique la bonne heure, elle me dit à nouveau qu'elle ferait mieux d'occuper le temps qui nous reste avec des choses insignifiantes.

Suite à son acte manqué il me vient de lui dire, en reprenant le verbe «to run » qu'elle a utilisé : "Unless you are running away from me, a capturing mother

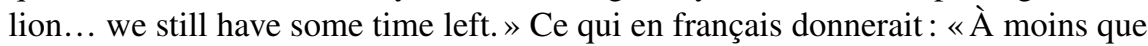
vous me fuyiez moi, une mère lionne qui capture... il nous reste encore du temps » Jessica se défend d'abord et me dit en prenant un ton de grande politesse, voire un peu mondain : «Mais je serais ravie que vous me disiez ce que vous pensez de mon rêve. » Puis elle regarde sa montre et se tait un moment manifestement troublée avant de poursuivre: «ça se pourrait que je vous fuis. Je suis tout le temps en train de courir et de fuir. Mon mari me l'a dit récemment. Je ne donne qu'une toute petite partie de moi et pour me protéger je crée souvent des écrans de fumée. »

Je voudrais reprendre cette séquence et, en particulier, mes deux interventions sous l'angle des considérations théoriques que je soulevais au départ. Ma première intervention semble assez proche du récit du rêve; je souligne le désir de Jessica de jouer et le sentiment qu'elle ne peut le faire qu'en cachette. Elle déstabilise toutefois l'analysante parce qu'elle aiguise les aspects transférentiels de ses mouvements et renforce leur extériorisation ${ }^{2}$. Si par son énoncé mon intervention peut éventuellement soutenir son désir de jouer et constituer une ouverture à une scène infantile, je la propose alors même que je représente - poursuivons cette hypothèse- un objet transférentiel menaçant, la mère lionne qu'elle préférerait voir dormir. Par le fait même que je parle et que je nomme son désir, je pourrais représenter une lionne qui a maintenant ouvert un œil ${ }^{3}$. Pour revenir à soit la manière dont nos interprétations font travailler l'écart ou la superposition de l'objet interne et de l'analyste objet «extérieur», l'un des points que je me proposais de faire travailler, cette première intervention interprétative encourage la superposition entre la réalité externe et la réalité interne et sollicite ainsi le déploiement dans le transfert de la réalité psychique de l'analysante.

Considérons maintenant la seconde interprétation. Elle a la particularité de se référer directement à la répétition agie ${ }^{4}$. Donnet (1995), qui a beaucoup travaillé la question de «l'agieren», considère que dans ces occurrences, l'interprétation permet de mobiliser au mieux la charge pulsionnelle dont le fantasme est investi. Ses remarques s'avèrent d'autant plus pertinentes que l'analysant manque à organiser une névrose de transfert comme ici Jessica.

«Si la cure est bien avant tout une analyse du transfert, l'enjeu de l'interprétation doit être maximal lorsqu'elle rencontre la répétition-agie-de transfert. Cette rencontre spécifie l'acmé de l' «action» analytique; c'est elle qui réalise l'optimisation de l'écart économico-symbolique, la liaison affects-représentation idoine, propre à l'établissement de cette «conviction» que Freud met au cœur de l'effet de vérité.» (Donnet, 1995, 174) 
Dans cette séquence je choisis d'intervenir au moment où l'affect d'angoisse est le plus présent, plus précisément le moment où il est, me semble-t-il, suffisamment proche de la représentation inconsciente déplacée sur moi, ce qui la pousse à une répétition agie, la réactualisation dans la séance de sa conflictualité (Jessica regarde sa montre, se trompe...). Il est banal de dire qu'au moment où s'actualise ainsi sa réalité psychique, Jessica la méconnaît. Par mon interprétation au point d'actualisation transférentielle, je mets en mots sa répétition agie en la reliant à son rêve. Plus précisément, je tente de remettre en jeu, en elle et entre nous, ce qui s'est joué dans le rêve mais qui, depuis le début de la séance restait hors jeu, en actes à défaut de pouvoir trouver une place dans le discours de Jessica. Je considère en effet que ce qu'il y a d'agi comporte un élément à mettre en jeu et que l'interprétation soutient cette mise en jeu. Dans cette perspective le renoncement et le dégagement suivront. Je rejoins Diatkine (1982) pour lequel :

«l'interprétation «idéale» est probablement celle qui peut se formuler quand les processus inconscients ont perdu leur impérieuse nécessité. » $(1982,733)$

La seconde illustration clinique nous permettra toutefois d'apprécier dans quelle mesure la mise en jeu de la réalité psychique dans la séance engage tout de même une certaine forme de renoncement dans le sens d'un lâcher prise. À travers cette séquence je voudrais aussi traiter de l'alternance de mouvements d'intériorisation et d'extériorisation, ou d'actualisation, dans le travail analytique à laquelle participe, évidemment, le travail interprétatif de l'analyste et ses aléas.

\section{«Et voilà qu'elle dort!»}

Samantha est une femme d'âge mûr avec laquelle je me suis très souvent sentie confuse au cours des premières années de notre travail. Longtemps, j'ai eu le sentiment curieux que son discours ne m'était pas adressé ou même qu'il n'était adressé à personne. La quatrième année de la cure a marqué un changement notable à ce niveau ${ }^{5}$. La séquence que je décris ici suit ce changement.

À la première séance après l'été, Samantha me dit que la reprise de l'analyse la prend de court, elle n'y a pensé qu'hier. Dans le cours de son récit des vacances passées avec des amis elle évoque Sarah, une jeune femme handicapée, à laquelle elle a peur de ressembler par sa léthargie et sa dépendance. J'entends qu'elle me parle d'un aspect d'elle-même et je choisis de laisser sa réalité psychique se déployer sans intervenir.

Le lendemain, Samantha me dit qu'elle se sent mal; elle se relâche. Elle ressent quelque chose d'émotionnel à l'idée de venir en analyse; elle s'en réjouit mais en même temps ça l'énerve. Son manque d'autonomie l'énerve: «J'ai un besoin féroce d'autonomie ou j'ai tellement peur du contraire.» Elle me dit que c'est en lien avec Sarah aussi et elle pleure. Elle enchaîne sur le manque de disponibilité de son mari ces temps-ci, puis sur une chute après la séance précédente. Ses 
chaussures ne lui tiennent pas aux pieds et elle en a marre de faire les choses comme il le faut. D'ailleurs avant de venir à sa séance hier elle a fait brûler une casserole. Elle ajoute, «voilà l'encadrement de la séance hier!»

Moi : Alors c'est moi qui vous encadre mal... (D'emblée je me dis que cette intervention est trop hâtive et surtout, insatisfaisante dans sa forme, "prête à porter», comme les chaussures mal adaptées aux pieds de ma patiente. J'ai l'impression d'avoir ré-agi comme si j' avais aussi une casserole sur le feu. Pendant toute la séance je note que quelque chose de cette précipitation perdure.)

Mon interprétation amène l'analysante à centrer ses propos sur la scène de l'analyse : «Et je n'ai plus d'argent, c'est le stress. Ça me crée une dualité par rapport à l'analyse. Je rêve de faire un bilan. Mais je suis paresseuse et je me remets à vous. Mon élan c'est de me laisser prendre en charge. Il n'y a plus de grand mystère à découvrir dans l'analyse. Alors au-delà du mystère qu'est-ce que je peux encore faire ici? En fait, je vois le bout mais est-ce que je me fourvoie en pensant cela? Le meilleur test serait l'abandon... (Petit silence) Ah ! j'ai rêvé cette nuit. Il y avait vous dans le rêve, comme souvent dans les rêves autour de la rentrée. C'était un rêve à moitié intime. Il y avait aussi M. X un personnage que je trouve émouvant. Dans ce rêve quelque chose m'affecte physiquement rien que d'y penser. J'étais dans l'appartement où j'habitais avant, comme si j'y habitais, ou plutôt j'y étais retournée mais c'était tout vide. Il y avait un lit par terre. Et vous étiez dans la chambre à côté, vous dormiez. J'hésitais à vous réveiller, je ne me sentais pas très bien et je tournais en rond. Je me regarde dans la glace, je refais la raie au milieu. Ça ne se voit pas, puis ça se voit, puis pas, comme si j' avais une raie de quatre centimètres... pleine de cônes comme de la mousse pour protéger. C'était la personnification de mon malêtre. Puis je tourne en rond. Je vais voir où vous êtes, j'hésite à vous réveiller, je ne le fais pas. Je vois le lit sur le sol et me dis que je peux dormir là.... euh, tous ces cônes sur ma tête, tous alignés, un était tordu, coupé, abîmé... j'ai l'image que c'était vivant et douloureux. Ça doit être l'influence de ma chute hier. Dans le rêve je me voyais dans la glace et mon visage était asymétrique. Quand j'ai fait cette chute je me suis dit: «ah, j'en ai marre, il faut qu'on s'occupe de moi.»

Moi : Et voilà qu'elle dort !

Samantha: «Je vous ai mis accessible et je vous rends inaccessible... Mon mari était loin ce week-end, j'étais jalouse. Même mon chat ne rentre pas à la maison. Si je dois tirer la patte du chat, ça va pas....

Moi : «Si vous devez me réveiller pour que je comprenne que ça ne va pas...»

Samantha: «Oui ça va pas... je ne peux le faire qu'avec mon mari, mais là je n'ai pas le sentiment de le contraindre puisque c'est normal dans le couple. C'est marrant cette image de vous faire dormir, je ne pouvais pas vous réveiller. »

Moi : Si j'ai bien compris, ce n'est pas avec M. X que je dormais?

Elle rit. Non. La chambre était vide, il n'y avait que le lit et nous. Vous me tourniez le dos.

J'interromps ici le récit de la séance pour approfondir, comme précédemment, la question de l'interprétation. L'interprétation «Et voilà qu'elle dort!» est 
intéressante pour mon propos. Dans son contenu, cette interprétation, comme toute interprétation, est surdéterminée (Le Guen, 1962). Par exemple, et même si je n'y pense qu'après l'avoir énoncée, je sais qu'enfant Samantha a souvent retrouvé sa mère endormie. Mais plus que sur le contenu, c'est sur la forme de cette intervention que je voudrais m'arrêter et la mettre en regard avec l'interprétation précédente, «C'est alors moi qui vous encadre mal... » et son caractère trop défini, arrêté. Dans la seconde intervention, mon «elle» («Et voilà qu'elle dort!») fait écho au «on» de l'analysante («Il faut qu'on s'occupe de moi.»). Dans mon énoncé restent en effet indéterminées la place de laquelle je parle et celle que je nomme «elle». Ces aspects sont comme mis en suspens. Ma formulation maintient un indécidable entre fantasme et «réalité», entre l'objet subjectif et l'objet objectivement perçu; je tente d'ouvrir un espace de jeu favorable au déploiement de la réalité psychique de l'analysante.

Lorsque Samantha dit «il faut qu'on s'occupe de moi», j'entends par cette formulation impersonnelle (il faut, on) qu'elle se trouve là en difficulté de subjectivation, ce que reflète sa formulation reflète. Si j'avais été plus précise et que je lui avais par exemple dit «Et voilà que je dors » ou que je l'avais renvoyée à sa mère, ou encore si je lui avais interprété ce à quoi elle arrive seule par la suite, j'aurais court-circuité le temps de la subjectivation. Par le «Et voilà qu'elle dort !», je souligne un aspect de son rêve mais je lui laisse le choix et le soin de s'en saisir; je la laisse libre de son mouvement d'intériorisation. En paraphrasant Winnicott qui compare l'interprétation à la spatule brillante de la situation établie, mon intervention fait briller un élément de son rêve et ainsi révèle ou renforce un potentiel de jeu. Samantha s'en saisit et en fait, elle-même, une interprétation : «je vous ai mis accessible et vous rends inaccessible». De fait, cette séquence nous amène à considérer aussi les interprétations faites par les patients eux-mêmes et dont Strachey dit qu'elles peuvent, elles aussi, être mutatives.

Dans la suite de la séance, à la faveur de ce mouvement de subjectivation et des associations de Samantha, mes interventions ( si vous devez me réveiller pour que je comprenne que ça ne va pas... » et « Si j'ai bien compris ce n'est pas avec M. $\mathrm{X}$ que je dormais. ») semblent prolonger assez naturellement le «Et voilà qu'elle dort» et permettre de l'inscrire dans des fantasmes originaires et dans une configuration oedipienne inversée.

À travers ces séquences cliniques j'ai cherché à rendre compte de la complexité du travail d'interprétation dont chaque forme ponctue, de manière singulière, l'analyse. Les particularités et les enjeux que j'ai dégagés ici correspondent à diverses questions qui ont surgi en moi au cours de ces cures où le travail analytique n'a pas été de soi, où il a même été à construire et où, corrélativement, j'ai eu longtemps à redécouvrir ou réinventer une fonction d'analyste, voire mon identité d'analyste, à travers mon mode de présence, mon écoute et mes interventions.

En reprenant les concepts que fait notamment travailler Donnet, je suggère que l'interprétation de transfert aide généralement à la mise en jeu de la réalité 
psychique et au déploiement du jeu analytique et peut, si nécessaire, contribuer à le complexifier. L'interprétation de transfert prolonge l'indécidable du transfert le temps nécessaire pour que la réalité psychique de l'analysant puisse suffisamment se déplier. J'ai cherché à rendre compte de la pertinence et des limites de telles interventions. En contrepoint, par le renoncement qu'elle promeut l'interprétation du transfert marque davantage une césure. Les subtilités de la réalité psychique et $\mathrm{du}$ jeu analytique nous renvoient toutefois immanquablement aux limites de considérations aussi réductrices.

nathalie zilkha 5, ch. malombré, 1206 genève, suisse

\section{Notes}

1. L'analyse se déroule en anglais.

2. D'autant plus que chargée d'une certaine irritation, elle prend un caractère un peu surmoïque.

3. Je laisse de côté ici la question du double figurée dans la présence des deux fillettes qui jouent ainsi que dans celle, ambiguë ou complice, des policiers. D'ailleurs Jessica elle-même aurait peut-être préféré rester endormie que de se réveiller pour la séance.

4. Je dirai même qu'elle la porte. En effet, dans ma formulation même, «moi, une mère lionne qui capture » je me désigne confondue avec l'objet de transfert. La question de savoir dans quelle mesure j'ai pu moi-même être, en partie, prise par l'actualisation transférentielle reste ouverte.

5. J'en ai rendu compte dans un travail antérieur, La dépendance, une réalité psychique ?, Rev. franç. psychanal., 2/2004, 495-507.

\section{Références bibliographiques}

Anzieu D., 1970, Eléments d'une théorie de l'interprétation, Rev. franç. psychanal., 34, ns 5-6, 755-820.

Diatkine R., 1983, Introduction à une discussion sur l'interprétation, Rev. franç. psychanal., 47, no 3,717-734.

Donnet J.-L.,1995, Le divan bien tempéré, Paris, PUF.

Freud S.,1914, Remémoration, répétition et perlaboration, OCP XII, 185-196.

Le Guen C., 1982, La dialectique freudienne 1. Pratique de la méthode psychanalytique, Paris, PUF.

M’Uzan de, M.,2004, Pour une cohérence théorique et doctrinale de l'interprétation, entretien avec M.-F. LavalHygonenq, in: Interpréter le transfert, Monographie de la rfp, Paris, PUF. 39-52

Strachey J.,1934, The nature of the therapeutic action of psychoanalysis, Intern. Journal of psychoanalysis, 15, 137159. Trad. franc. par C. David. La nature de l'action thérapeutique de la psychanalyse, in: Interprétation I. Un processus mutatif, Monographie de la rfp, Paris: PUF, 1999, 33-64.

Winnicott D.W., 1971, Playing and Reality, London, Tavistock. Trad. Franç. Par C. Monod et J.-B. Pontalis. 